\title{
On the singular values decoupling in the Singular Spectrum Analysis of volcanic tremor at Stromboli
}

\author{
R. Carniel ${ }^{1}$, F. Barazza ${ }^{1}$, M. Tárraga ${ }^{2}$, and R. Ortiz ${ }^{2}$ \\ ${ }^{1}$ Dipartimento di Georisorse e Territorio, Università di Udine, 33100 Udine, Italy \\ ${ }^{2}$ Departamento de Volcanología, Museo Nacional de Ciencias Naturales, CSIC, Madrid, Spain
}

Received: 6 December 2005 - Revised: 11 October 2006 - Accepted: 11 October 2006 - Published: 16 October 2006

\begin{abstract}
The well known strombolian activity at Stromboli volcano is occasionally interrupted by rarer episodes of paroxysmal activity which can lead to considerable hazard for Stromboli inhabitants and tourists. On 5 April 2003 a powerful explosion, which can be compared in size with the latest one of 1930, covered with bombs a good part of the normally tourist-accessible summit area. This explosion was not forecasted, although the island was by then effectively monitored by a dense deployment of instruments. After having tackled in a previous paper the problem of highlighting the timescale of preparation of this event, we investigate here the possibility of highlighting precursors in the volcanic tremor continuously recorded by a short period summit seismic station. We show that a promising candidate is found by examining the degree of coupling between successive singular values that result from the Singular Spectrum Analysis of the raw seismic data. We suggest therefore that possible anomalies in the time evolution of this parameter could be indicators of volcano instability to be taken into account e.g. in a bayesian eruptive scenario evaluator. Obviously, further (and possibly forward) testing on other cases is needed to confirm the usefulness of this parameter.
\end{abstract}

\section{Introduction}

Stromboli is the prototype for strombolian activity, observed since at least 3-7 A.D. (Rosi et al., 2000). Occasionally, paroxysmal phases are observed (Barberi et al., 1993; Jaquet and Carniel, 2003) involving an additional kind of magma, low-porphyritic and volatile-rich (Francalanci et al., 2004) that apparently plays a major role in the development of such phases. This involvement of a different magma suggests that there may be a sufficiently long timescale of preparation for

Correspondence to: R. Carniel

(roberto.carniel@uniud.it) this kind of events, and therefore the possible appearance of recordable precursors. Talking about timescales, (Ripepe et al., 2002) identified two degassing and explosive regimes at Stromboli, linked to the fresh gas-rich magma supply rate, that alternate on a 5-40 min timescale. At a longer timescale, (Carniel and Di Cecca, 1999) identified days-to-weeks-long dynamical phases, sometimes separated by abrupt transitions. Talking about possible precursors, (Carniel and Iacop, 1996b) showed that paroxysmal phases are sometimes preceded by increasing lower frequency content in the tremor. Due to the complexity of the physical processes involved, a stochastic (Jaquet and Carniel, 2001, 2003) or dynamical approach (Carniel and Di Cecca, 1999; Carniel et al., 2003) is often a more appropriate choice for short-to-medium term forecasts aimed e.g. to schedule the tourist excursions when volcanic hazard is lower.

On 28 December 2002, an effusive eruption (Alean et al., 2006) marked the most significant effusive episode since 1985-1986. This induced considerable morphological changes before its end on 21-22 July 2003 (Calvari, 2003) and showed the most energetic associated explosive event at 07:12 GMT of 5 April 2003. The event started with a reddish ash emission related to collapses within the craters, soon replaced by darker juvenile material from NE Crater and followed by similar emissions from SW Crater which mushroom-shaped dark cloud rose about $1 \mathrm{~km}$ above the summit (Calvari, 2003). Bombs, ash, and blocks affected most the Western sector and the village of Ginostra; the volcano top above $700 \mathrm{~m}$ a.m.s.l. was completely covered by bombs, including meter-sized ones (Alean et al., 2006), and scientific equipment, including the seismic station of the University of Udine, was damaged or destroyed. In a previous paper, Carniel et al. (2006) tackled the problem of highlighting the timescale of preparation of this event, showing that the volcanic tremor recorded continuously by a short period seismic station is sufficiently informative to derive this kind of information. In fact, non-stationarity is a key

Published by Copernicus GmbH on behalf of the European Geosciences Union. 
characteristic of the tremor (Konstantinou and Schlindwein, 2002), as it offers the possibility of monitoring changes in parameters derived from an experimental time series and their possible use in forecasting (Carniel and Di Cecca, 1999) or at least to highlight different regimes (Ripepe et al., 2002; Carniel et al., 2003; Harris et al., 2005; Jones et al., 2005). By the use of spectral and dynamical analysis (Carniel et al., 2006) showed that the paroxysmal phase of 5 April 2003 have built up over at least the previous 2.5 hours, but the application of the Material Failure Forecast Method during the days before the event revealed a consistent trend that suggests a preparation of the paroxysm further in the past, an evolution which was possibly temporarily "paused" the day before, before finally accelerating during the consistent dynamical phase of the last few hours. In any case, there is sufficient "persistence" in the data to look for some kind of precursor as a hind-cast exercise. In particular, the Singular Spectrum Analysis is the methodology that shows the most promising potential in the search for precursors. We therefore start by presenting this technique in further detail.

\section{The Singular Spectrum Analysis (SSA) technique}

Time series analysis has recently profited from the application of spectral decomposition of matrices (e.g. (Marelli et al., 2002; Mineva and Popivanov, 1996; Pereira and Maciel, 2001)), which has its roots mostly in chaos theory (Takens, 1981; Broomhead and King, 1981). The SSA consists of several steps (Golyandina et al., 2001; Aldrich and Barkhuizen, 2003). In the first step (embedding), the one dimensional time series is recast as an $L$-dimensional time series (trajectory matrix). In the second step (singular value decomposition), the trajectory matrix is decomposed into a sum of orthogonal matrices of rank one. These two steps constitute the decomposition stage of SSA. In the third and fourth steps, the components are grouped and the time series associated with the groups are reconstructed.

\subsection{Decomposition stage}

\subsubsection{Embedding of time series}

Embedding expands the original time series into what is referred to as trajectory matrix of the system. This matrix is associated with a certain window length that is the embedding dimension. The series is expanded by giving it a unitary lag and creating a certain number of lagged vectors. So, for the time series $\mathbf{x}=\left\{x_{1}, x_{2}, \ldots, x_{n}\right\}$ and for an embedding dimension $m$, we obtain the trajectory matrix:

$$
\mathbf{X}=\left\{\begin{array}{cccc}
x_{1} & x_{2} & \ldots & x_{m} \\
x_{2} & x_{3} & \ldots & x_{m+1} \\
\vdots & \vdots & \ddots & \vdots \\
x_{n-m+1} & x_{n-m+2} & \ldots & x_{n}
\end{array}\right\}
$$

This is by definition a Hankel matrix, as it is $\mathbf{X}_{i, j}=\mathbf{x}_{i+j-1}$. The size of the embedding window $m$ (number of columns of the trajectory matrix) should be sufficiently large to capture the global behavior of the system. A common method to determine $m$ is to use the first zero of the linear correlation between the first and the last column of the trajectory matrix. Another method used to determine $m$ (Shaw, 2000) uses the point where mutual information between the first and the last column of the trajectory matrix reaches the first minimum (Fraser, 1986; Fraser and Swinney, 1986). This is in principle a better choice, since it takes into account also the non-linear correlation within the time series.

\subsubsection{Decomposition of time series}

Once the time series are embedded into the trajectory matrix $\mathbf{X}$, the singular value decomposition is performed on such matrix. The singular values decomposition technique allows to obtain the decomposition:

$$
\mathbf{X}=\mathbf{U S} \mathbf{V}^{T}
$$

where $\mathbf{U}$ is a $(n-m+1) \times(n-m+1)$ real orthogonal matrix, $\mathbf{V}$ is a $m \times m$ real orthogonal matrix and $\mathbf{S}$ is a $(n-m+1) \times m$ diagonal real matrix such that its elements are the singular values of the trajectory matrix $\mathbf{X}$. This is done by computing the lagged covariance matrix

$$
\mathbf{C}=\mathbf{X}^{T} \mathbf{X}
$$

so $\mathbf{C}$ is a symmetric semidefinite positive matrix and then a unique spectral decomposition exists:

$$
\mathbf{C}=\Phi \Lambda \Phi^{T}
$$

where $\Phi$ is a real orthonormal matrix such that its columns are the eigenvectors of $\mathbf{C}$, and $\Lambda$ is a real diagonal matrix such that its elements $\sigma_{i}^{2}$ are the eigenvalues of $\mathbf{C}$ in decreasing order. One can now observe that

$$
\begin{aligned}
\mathbf{C} & =\mathbf{X}^{T} \mathbf{X} \\
& =\left(\mathbf{U S V}^{T}\right)^{T}\left(\mathbf{U S V}^{T}\right) \\
& =\mathbf{V S}^{T} \mathbf{U}^{T} \mathbf{U S V}^{T}
\end{aligned}
$$

but $\mathbf{U}$ is an orthonormal matrix and $\mathbf{S}$ is a diagonal matrix, so $\mathbf{U}^{T} \mathbf{U}=\mathbf{I}$ and $\mathbf{S}^{T} \mathbf{S}=\mathbf{S}^{2}$. Therefore

$$
\mathbf{C}=\mathbf{V S}^{2} \mathbf{V}^{T}
$$

Comparing Eqs. (3) and (4) one obtains that

$$
\begin{aligned}
\Phi & =\mathbf{V} \\
\Lambda & =\mathbf{S}^{2}
\end{aligned}
$$

So one can deduce the important consequence that the right eigenvectors of $\mathbf{X}$ are the eigenvectors of $\mathbf{C}$ while the singular values of $\mathbf{X}$ are the square root of the eigenvalues of $\mathbf{C}$. 
Therefore:

$$
\mathbf{S}=\left\{\begin{array}{cccc}
\sigma_{1} & 0 & \ldots & 0 \\
0 & \sigma_{2} & \ldots & 0 \\
\vdots & \vdots & \ddots & \vdots \\
0 & 0 & \ldots & \sigma_{m} \\
0 & 0 & \ldots & 0 \\
\vdots & \vdots & \ddots & \vdots \\
0 & 0 & \ldots & 0
\end{array}\right\}, \sigma_{1} \geq \sigma_{2} \geq \ldots \geq \sigma_{m} \geq 0
$$

Another way to write Eq. (1) is the so called spectral decomposition:

$$
\mathbf{X}=\sum_{i=1}^{m} \sigma_{i} \mathbf{u}_{i} \mathbf{v}_{i}^{T}
$$

where $\mathbf{u}_{i}$ and $\mathbf{v}_{i}$ are respectively U's and V's matrix i-th columns. But we had demonstrated that $\mathbf{V}=\Phi$, and from Eq. (1) it is not difficult to show that $\mathbf{X V}=\mathbf{U S}$. So $\mathbf{v}_{i}=\Phi_{i}$ and $\sigma_{i} \mathbf{u}_{i}=\mathbf{X} \Phi_{i}$ where $\Phi_{i}$ is $\Phi$ 's i-th column. Substituting this equality in Eq. (5) permits us to obtain a simpler and more useful form for X's decomposition:

$$
\mathbf{X}=\sum_{i=1}^{m} \mathbf{X} \Phi_{i} \Phi_{i}^{T}
$$

\subsection{Reconstruction stage}

The aim of this stage is to separate the additive components of the time series. It can be seen as separating the time series into two groups: "our signal" and the "noisy" components, which are by definition the components we are not interested in.

The idea is to project the trajectory matrix over a $q$ dimensional space. In fact, in Eq. (5), every term of the sum has a lower importance with respect to the previous one in the construction of the signal. Such importance is given by the weight $\sigma_{i}$ of each singular value of the base and $\sigma_{1} \geq \sigma_{2} \geq \ldots \geq \sigma_{m} \geq 0$ by construction. Then we can approximate Eq. (5) and (6) with the following:

$$
\mathbf{X}_{\mathbf{q}}=\sum_{i=1}^{q} \sigma_{i} \mathbf{u}_{i} \mathbf{v}_{i}^{T}
$$

and

$$
\text { noise }=\sum_{i=q+1}^{m} \sigma_{i} \mathbf{u}_{i} \mathbf{v}_{i}^{T}
$$

or, in a simpler form:

$$
\mathbf{X}_{\mathbf{q}}=\sum_{i=1}^{q} \mathbf{X} \Phi_{i} \Phi_{i}^{T}
$$

and

$$
\text { noise }=\sum_{i=q+1}^{m} \mathbf{X} \Phi_{i} \Phi_{i}^{T}
$$

The criteria for this separation are not completely formalized and they depend on knowledge of the data, and obviously on $\sigma_{i}$ 's modules. For example if the first $q$ singular values are much greater than the others, then the choice can be straightforward.

The new matrix $\mathbf{X}_{\mathbf{q}}$ is not always a trajectory matrix (because in general it is not a Hankel matrix) and then it does not directly represent the noise-free $\mathbf{x}$ time series. However this time series can be obtained by a diagonal average method. For example, if $\mathbf{X}_{\mathbf{q}}$ is the matrix:

$$
\mathbf{X}_{\mathbf{q}}=\left\{\begin{array}{lll}
x_{1,1} & x_{1,2} & x_{1,3} \\
x_{2,1} & x_{2,2} & x_{2,3} \\
x_{3,1} & x_{3,2} & x_{3,3} \\
x_{4,1} & x_{4,2} & x_{4,3}
\end{array}\right\}
$$

then we obtain:

$$
\begin{aligned}
\mathbf{x}_{\mathbf{q}}= & \left\{x_{1,1}, \frac{x_{2,1}+x_{1,2}}{2}, \frac{x_{3,1}+x_{2,2}+x_{1,3}}{3},\right. \\
& \left.\frac{x_{4,1}+x_{3,2}+x_{2,3}}{3}, \frac{x_{4,2}+x_{3,3}}{2}, x_{4,3}\right\}
\end{aligned}
$$

In conclusion, the noise-free time series $\mathbf{x}_{\mathbf{q}}$ depend on the choice of the parameters $m$ and $q$, so we can write:

$$
\mathbf{x}_{\mathbf{q}}=S S A(\mathbf{x} ; m, q)
$$

Obviously, the level of de-noising is higher when $q$ is lower. (Hegger et al., 1999) suggest that $q$ should be at least the correct embedding dimension, and $m$ considerably larger (e.g. $m=2 q$ or larger).

\section{Coupling of the singular values}

The behavior of the singular values (also called the spectrum of the singular values) can contain useful indications on the composition of the signal (i.e. Yang and Tse, 2003). In particular, for a sufficiently high embedding dimension some of the singular values tend to show a certain degree of coupling, that is $\sigma_{2} \rightarrow \sigma_{1}, \sigma_{4} \rightarrow \sigma_{3}$ and so on. An example is shown in Fig. 1. Note that in Fig. 1, the singular values are computed using the following definition of the covariance matrix:

$$
\mathbf{C}=\frac{1}{n-m} \mathbf{X}^{T} \mathbf{X}
$$

In this way the singular values are detrended, allowing a simpler visualization. 


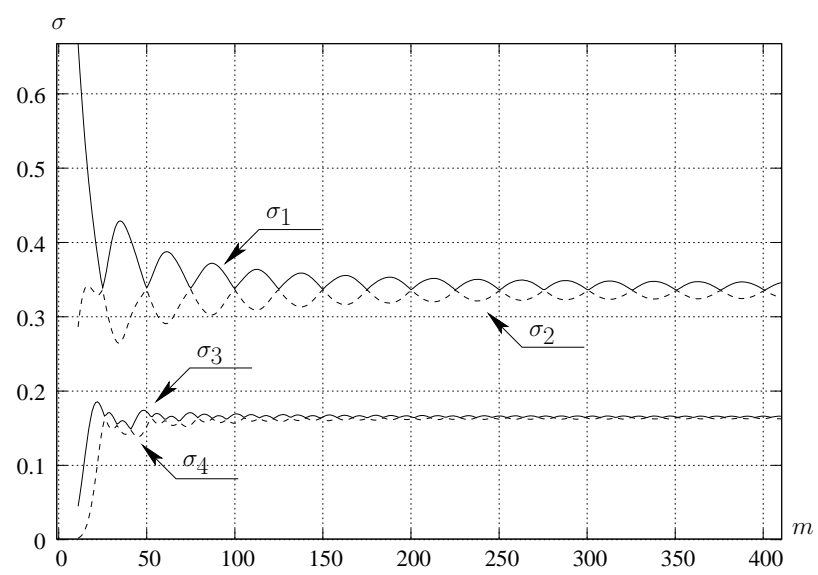

Fig. 1. Example of coupling between singular values for the signal $\sin (20 \cdot 2 \pi t)+0.7 \sin (57 \cdot 2 \pi t)$ (sampled at $1000 \mathrm{~Hz}$ ) in an increasing embedding dimension $m=10$ to 400 .

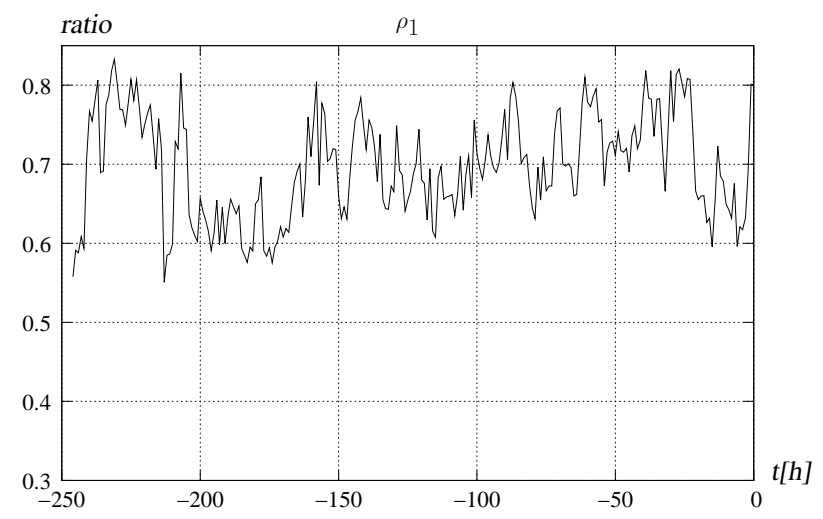

Fig. 2. $\rho_{1}=\frac{\sigma_{2}}{\sigma_{1}}$ in an embedding space of dimension $m=10$, for Stromboli volcanic tremor.

We can define the "degree of coupling" as:

$$
\begin{aligned}
\rho_{1} & =\frac{\sigma_{2}}{\sigma_{1}} \\
\rho_{2} & =\frac{\sigma_{4}}{\sigma_{3}} \\
& =\ldots \\
\rho_{k} & =\frac{\sigma_{2 k}}{\sigma_{2 k-1}}
\end{aligned}
$$

\section{The data}

One of the longest seismic time series available at Stromboli comes from an automatic station installed in 1989 by the Dipartimento di Georisorse e Territorio of the University of Udine (Beinat et al., 1994) with the purpose of studying the long-term evolution of the strombolian activity (Carniel and Iacop, 1996a). The summit station, based on
3 Willmore MKIII/A seismometer $\left(f_{0}=0.5 \mathrm{~Hz}\right)$, is located at $800 \mathrm{~m}$ a.m.s.l. and at about $300 \mathrm{~m}$ from the craters (Beinat et al., 1994). During this last effusive phase, the hardware and software of the receiving station was upgraded in collaboration with CSIC, Madrid, for a continuous acquisition and internet data transmission. Data are now sampled with 16 bits $(96 \mathrm{~dB})$ at $50 \mathrm{~Hz}$ (Ortiz et al., 2001; Carniel et al., 2006). Although at the time of 5 April 2003 paroxysmal event a dense network of monitoring equipment installed by the Civil Defence was operating, the explosion was not forecasted by any evident change in the data. In the following we will examine with the SSA methodology described above the seismic data recorded by our station during the days before this paroxysmal event, in particular from 25 March to 5 April 2003. The confidence in the method of analysis goes back again to Carniel et al. (2006), where another promising parameter was studied, derived from the SSA methodology:

$$
r_{k}=\frac{\sum_{i=1}^{k} \sigma_{i}}{\sum_{i=k+1}^{m} \sigma_{i}}
$$

The idea behind this parameter was to monitor the time evolution of the relative weight of the first $k$ SSA components in the construction of the full tremor signal.

\section{From tremor to singular values coupling}

First of all we subdivide the raw data in windows of $60 \mathrm{~s}$ (i.e., 3000 sample points at $50 \mathrm{~Hz}$ ). Both the methods of autocorrelation function and of mutual information (see Sect. 2.1.1) supply an embedding dimension estimate $m$ between 7 and 10 , so $m=10$ seems a correct minimal choice for the construction of the trajectory matrix. The singular values are computed on each of the one-minute time windows. In order to avoid amplitude-dependent effects, before the calculation of the singular values, the data in each time window is normalized to zero mean and unitary variance. We start our analysis with the minimum embedding dimension $m=10$.

\subsection{Behaviour of $\rho_{k}$ parameter for $m=10$}

We analyze the time evolution of $\rho_{k}$ approaching the paroxysmal event, that will be denoted by the hour "zero", at the extreme right of our graphs, for the embedding dimension $m=10$ (This embedding dimension is probably too small to capture the behaviour of the signal but allowed a very fast analysis). The first two singular values do not show any anomalous behaviour in their ratio $\rho_{1}$, as can be seen in Fig. 2. However, if we plot the same time evolution but we take into account the "degree of coupling" between the singular values $\sigma_{3}$ and $\sigma_{4}$, i.e. $\rho_{2}$, we observe a very clear anomalous decrease of the parameter, that starts to diverge 


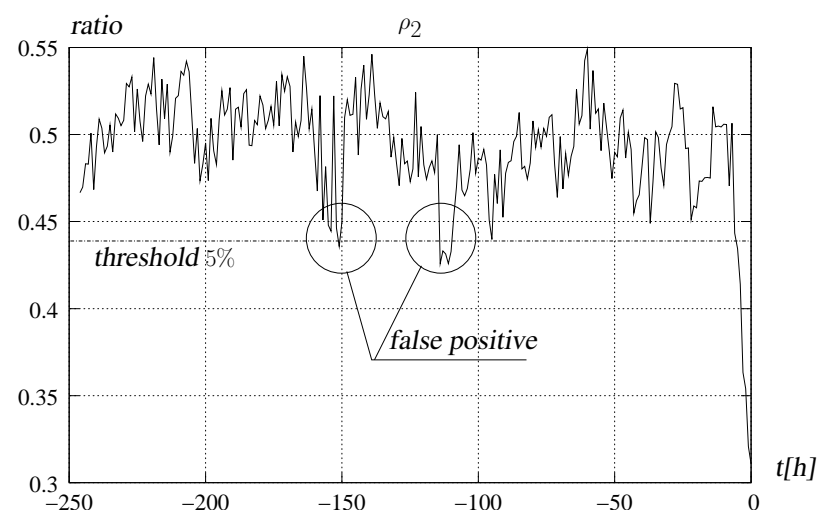

Fig. 3. $\rho_{2}=\frac{\sigma_{4}}{\sigma_{3}}$ in an embedding space of dimension $m=10$, for Stromboli volcanic tremor.

from the "normal" value - that theoretically, as we have shown, should be close to unity - already about $7 \mathrm{~h}$ before the explosion (Fig. 3). Note that it is important to observe the relative change in the behaviour of the $\rho_{k}$ rather than the absolute change. In fact, the coupling is observed more easily for singular values of smaller indexes, while the phenomenon tends to be less evident as the index is increased. Moreover, the physical explanation of the coupling is still subject of research.

If we assume the lowest $5 \%$ percentile of the coupling parameter range as a threshold for an alert, in this case the absolute value of the threshold is about 0.45 . This threshold generates two short-lasting false alerts at about 115 and $152 \mathrm{~h}$ before the explosion, but gives a "warning time" before the real explosion of more than 6 hours

\subsection{Behaviour of $\rho_{k}$ parameter for $m>10$}

In order to verify that the anomalous behaviour is not strictly dependent on the particular choice of the embedding dimension $m=10$, we performed a similar analysis also in higher dimensions, in particular for $m=20$ and $m=100$. In both cases we observe again that some of the "degree of coupling" ratios show a strong decrease approaching the paroxysm. Figure 4 shows for instance the anomalous time behaviour of the ratio $\rho_{3}=\frac{\sigma_{6}}{\sigma_{5}}$ in the embedding dimension $m=20$, while Fig. 5 shows the anomalous decrease of $\rho_{15}=\frac{\sigma_{30}}{\sigma_{29}}$ in an embedding with $m=100$.

Using again the 5\% percentile criterion, for $m=20$ we have a threshold of about 0.75 . As for the case $m=10$, we obtain false alerts at about 115 and $152 \mathrm{~h}$ before the true explosion. Also in this case however, the explosion is preceded by a warning more than $6 \mathrm{~h}$ in advance.

For $m=100$ the $5 \%$ percentile threshold corresponds to an absolute value of about 0.89 . Also in this case we have false alerts at about 115 and $152 \mathrm{~h}$ before the true explosion, and an additional one about $48 \mathrm{~h}$ before the explosion.

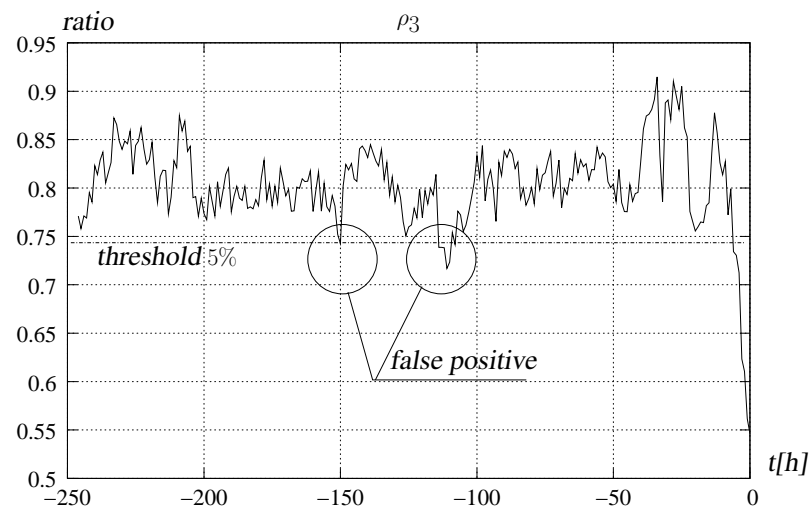

Fig. 4. $\rho_{3}=\frac{\sigma_{6}}{\sigma_{5}}$ in an embedding space of dimension $m=20$, for Stromboli volcanic tremor.

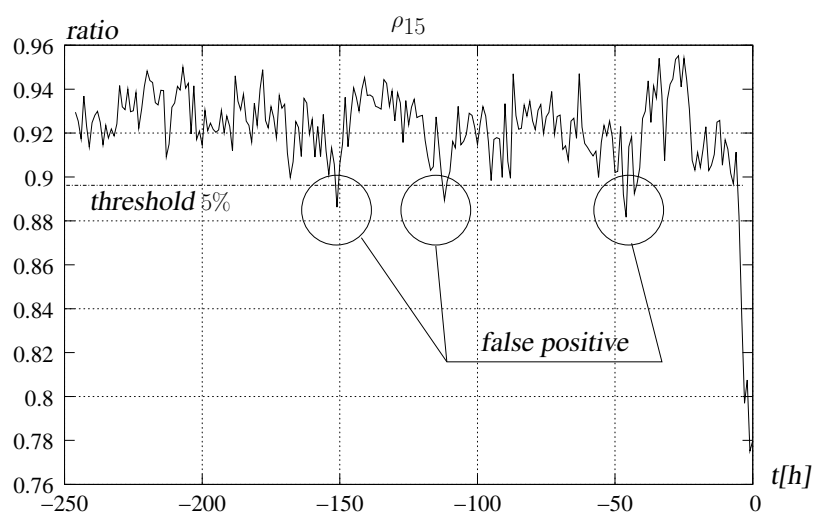

Fig. 5. $\rho_{15}=\frac{\sigma_{30}}{\sigma_{29}}$ in an embedding space of dimension $m=100$, for Stromboli volcanic tremor.

\section{Practical issues on the use of the $\rho_{k}$ parameter}

Close to the paroxysmal event, as we have shown, only some of the $\rho_{k}$ parameters show an anomalous decreasing behaviour. Other $\rho_{k}$ on the contrary don't show anything interesting. Consequently, a problem arises regarding the number of parameters to be monitored in order to observe possible anomalous behavior. We propose here a solution to this problem, with the definition of a "summarizing" parameter defined as the minimum of all $\rho_{k}$ computed in a given embedding dimension. Care should be taken however in order to obtain meaningful results: the last ratios should be excluded from this minimum calculation. The last (i.e. the least important) components of the SSA decomposition are in fact associated to what is essentially a noise signal (8), and should therefore be removed. We can then finally define:

$$
\hat{\rho}_{q}=\min _{k=1 . . q}\left\{\rho_{k}-\mu_{k}\right\} \quad q<m
$$

where $\mu_{k}$ is the average of the ratio $\rho_{k}$ that can be estimated during a normal period of activity (in our case the first 4 days 
$\hat{\rho}_{4}$

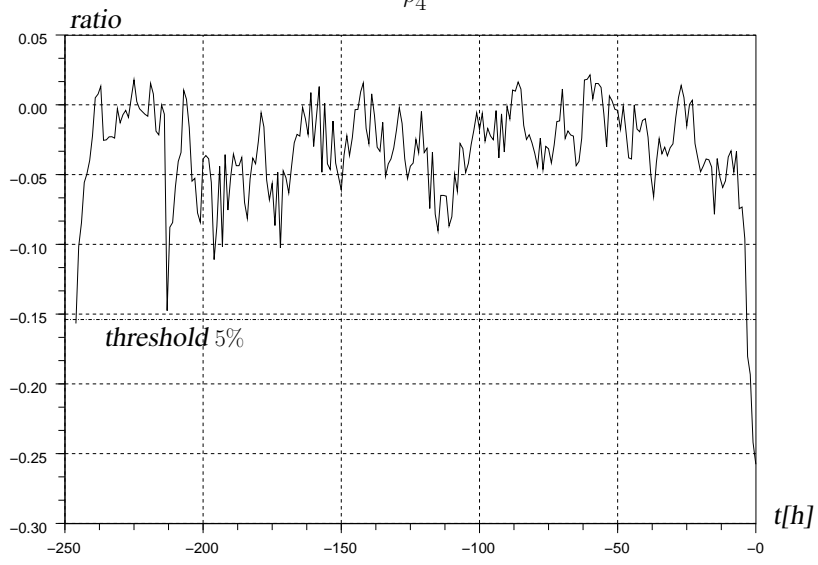

Fig. 6. $\hat{\rho}_{4}$ in an embedding space of dimension $m=20$, for Stromboli volcanic tremor.

in the graph). A suitable value of $q$ should be chosen; a rule of thumb is to take

$$
q \simeq \frac{1}{3} m
$$

In Fig. 6 the time evolution of the parameter $\hat{\rho}_{q}$ is shown for $q=4$ in an embedding dimension $m=20$. The decreasing anomalous behaviour is again very evident before the paroxysmal event.

In Fig. 4 a phase is evident with a peculiar behaviour starting about 40 hours before the explosion. This is the time at which Carniel et al. (2006) observe the first successful application of the Failure Forecast Method (FFM), that gives a forecast for 5 April 2003 at 15 GMT (see line 1 in Fig. 9 of Carniel et al. (2006)). More than $6 \mathrm{~h}$ before the explosion, the $\rho_{2}, \rho_{4}, \rho_{15}$ and $\hat{\rho}_{4}$ parameters (see Fig. 3, 4, 5 and 6, respectively) go below the percentile threshold of $5 \%$. At this time the FFM (see line 2 in Fig. 9 in Carniel et al. (2006)), after an apparent recovery of the stability, is able again to give a (even more reliable) forecast, for 5 April 2003 at 10:20 GMT, with a less than $3 \mathrm{~h}$ difference with respect to the real occurrence of the explosion.

A practical problem is that there are some embedding dimension ranges for which the anomalous behaviour disappears. One can think of a minimization procedure similar to the one adopted for the index of the ratio, but exploring e.g. every embedding dimension in the range $m=10$ to 500 , although theoretically possible, can lead to unacceptable computing times.

Also the choice of the minimum embedding dimension to use is not straightforward. The value suggested by the first zero of the autocorrelation function or by the first minimum of the mutual information (i.e. $m=10$ ) seems in fact too low.

\section{Conclusions}

Forecasting paroxysmal events such as the one recorded on Stromboli on 5 April 2003 is of paramount importance, especially at a volcano like Stromboli, where tourism is a major economical resource. This is undoubtly a difficult task if we consider the forecast in a deterministic sense. However, if we consider the risk of a volcano in a probabilistic sense (Aspinall et al., 2003, 2005) the issuing of a Temporary Increase in Probability of a paroxysmal event would be already a considerable success. In order to do this, a number of parameters should be monitored and their anomalies highlighted and weighted in a bayesian sense. Different useful parameters can be derived from the Singular Spectrum Analysis, one of which - the $r_{k}$ parameter weighting the relative importance of the first $k$ SSA components in the construction of a geophsyical signal - was proposed by Carniel et al. (2006). In this paper we have shown the potential of another family of parameters, $\rho_{k}$, which measure the degree of coupling between successive singular values. A practical way of getting rid of the problem of how to choose which of these couplings to monitor was also proposed, with the parameter $\hat{\rho}_{q}$, which looks for the (significant) minimum of these degrees of coupling. An important brand new class of monitoring parameters are therefore available for inclusion in bayesian eruptive scenario evaluators. Obviously, further (and possibly forward) testing on other cases is needed for testing the effective forecasting capabilities of these new parameters.

Acknowledgements. The methodologies developed and applied in this work are partially supported by the projects "V4 - Conception, verification and application of innovative techniques for studying volcanoes" by the Istituto Nazionale di Geofisica e Vulcanologia - Dipartimento Protezione Civile, Italy, "TEGETEIDE - Técnicas geofísicas y geodésicas para el estudio de la zona volcánica activa del complejo Teide - Pico Viejo (Tenerife)", Spain, TEIDEVS (Spain, CGL2004-05744), INGV-DPC V4 "Conception, verification, and application of innovative techniques to study active volcanoes" (Italy) and PRIN 2004131177 "Numerical and graphical methods for the analysis of time series data" (Italy). The SCILAB package (see http://www.scilab.org) was used to integrate the different analysis routines. Italian Civil Defence provided logistic support when access to the summit area was forbidden. The seismic station was originally installed under a research grant of the Italian Gruppo Nazionale di Vulcanologia. The authors gratefully acknowledge the collaboration of J. Alean, S. Ballarò, S. Calvari, C. Cardaci, M. Fulle, A. García, A. Llinares, P. Malisan, V. Perin, M. Ripepe and three anonymous referees in the various stages of data acquisition, logistic help, data analysis, interpretation and manuscript reviewing.

Edited by: J. Marti

Reviewed by: three referees 


\section{References}

Aldrich, C. and Barkhuizen, M.: Process system identification strategies based on the use of singular spectrum analysis, Minerals Engineering, 16, 815-826, 2003.

Alean, J., Carniel, R., and Fulle, M.: Stromboli online, volcanoes of the world: Information on Stromboli, Etna and other volcanoes, http://stromboli.net, 2006.

Aspinall, W., Woo, G., Voight, B., and Baxter, J.: Evidence-based volcanology: application to eruption crises, Journal of Volcanology and Geothermal Research, 128, 273-285, 2003.

Aspinall, W., Carniel, R., Hincks, T., Jaquet, O., and Woo, G.: Using Hidden Multi-state Markov models with multi-parameter volcanic data to provide empirical evidence for alert level decision-support, Journal of Volcanology and Geothermal Research, 153, 112-124, 2005.

Barberi, F., Rosi, M., and Sodi, A.: Volcanic hazard assessment at Stromboli based on review of historical data, Acta Vulcanologica, 3, 173-187, 1993.

Beinat, A., Carniel, R., and Iacop, F.: Seismic station of Stromboli: 3-component data acquisition system, Acta Vulcanologica, 5, 221-222, 1994.

Broomhead, D. and King, G.: Extracting qualitative dynamics from experimental data, Phisica, D(20), 217-236, 1981.

Calvari, S.: Stromboli report, Bulletin Global Volcanism Network, Smithsonian Institution, Smithsonian Institution, 28, 8, 5-5, 2003.

Carniel, R. and Di Cecca, M.: Dynamical tools for the analysis of long term evolution of volcanic tremor at Stromboli, Annali di Geofisica, 42, 3, 483-495, 1999.

Carniel, R. and Iacop, F.: On the persistency of crater assignment criteria for Stromboli explosion-quakes, Annali di Geofisica, 39, 2, 347-359, 1996a.

Carniel, R. and Iacop, F.: Spectral precursors of paroxysmal phases of Stromboli, Annali di Geofisica, 39, 2, 327-345, $1996 \mathrm{~b}$.

Carniel, R., Di Cecca, M., and Rouland, D.: Ambrym, Vanuatu (July-August 2000): Spectral and dynamical transitions on the hours-to-days timescale, Journal of Volcanology and Geothermal Research, 128, 1-3, 1-13, 2003.

Carniel, R., Ortiz, R., and Di Cecca, M.: Spectral and dynamical hints on the timescale of preparation of the 5 April 2003 explosion at Stromboli volcano, Can. J. Earth Sci. , 43, 41-55, 2006.

Francalanci, L., Tommasini, S., and Conticelli: The volcanic activity of Stromboli in the 1906-1998 AD period: mineralogical, geochemical and isotope data relevant to the understanding of the plumbing system, Journal of Volcanology and Geothermal Research, 131, 179-211, 2004.

Fraser, A.: Using mutual information to estimate metric entropy in Dimensions and Entropies in Chaotic Systems, Springer Verlag, 82-91, 1986.

Fraser, A. and Swinney, H.: Independent coordinates for strange attractors from mutual information, Phis.Rev. A., 33(2), 1134$1140,1986$.
Golyandina, N., Nekrutkin, V., and Zhigljavsky, A.: Analysis of time series structure-SSA and related techniques, Chapman \& Hall/CRC, 2001.

Harris, A., Carniel, R., and Jones, J.: Identification and Modelling of Variable Convective Regimes at Erta Ale lava lake, Journal of Volcanology and Geothermal Research, 142, 3-4, 207-223, 2005.

Hegger, R., Kantz, H., and Schreiber, T.: Practical implementation of nonlinear time series methods: The TISEAN package, CHAOS, 9, 413-435, 1999.

Jaquet, O. and Carniel, R.: Stochastic modelling at Stromboli: a volcano with remarkable memory, Journal of Volcanology and Geothermal Research, 105, 249-262, 2001.

Jaquet, O. and Carniel, R.: Multivariate stochastic modelling: towards forecasts of paroxysmal phases at Stromboli, Journal of Volcanology and Geothermal Research, 128, 1-3, 261-271, 2003.

Jones, J., Carniel, R., Malone, S., and Harris, A.: Seismic characteristics of variable convection at Erta Ale lava lake, Ethiopia, Journal of Volcanology and Geothermal Research, 153, 64-79, 2005.

Konstantinou, K. and Schlindwein, V.: Nature, wavefield properties and source mechanisms of volcanic tremor: a review, Journal of Volcanology and Geothermal Research, 119, 161-187, 2002.

Marelli, L., Bilato, R., Franz, P., Martin, P., Murai, A., and O'Gorman, M.: Singular spectrum analysis as a tool for plasma fluctuation analysis, Review of Scientific Instruments, 72(1), 499-502, 2002.

Mineva, A. and Popivanov, D.: Method for single-trial readiness potential identification, based on singular spectrum analysis, Journal of Neuroscience Method, 68, 91-99, 1996.

Ortiz, R., García, A., and Astiz, M.: Instrumentación en Volcanología, Servicio de Publicaciones del Cabildo Insular de Lanzarote, 345, 2001.

Pereira, W. and Maciel, C.: Performance of ultrasound echo decomposition using singular spectrum analysis, Ultrasound in Medicine and Biology, 27(9), 1231-1238, 2001.

Ripepe, M., Harris, A., and Carniel, R.: Thermal, seismic and infrasonic evidences of variable degassing rates at Stromboli volcano, Journal of Volcanology and Geothermal Research, 118, 285-297, 2002.

Rosi, M., Bertagnini, A., and Landi, P.: Onset of the persistent activity at Stromboli Volcano (Italy), Bulletin of Volcanology, 62, 294-300, 2000.

Shaw, R.: The Dripping Faucet as a Model Chaotic System, Aerial Press, Santa Cruz, CA., 2000.

Takens, F.: Detecting strange attractor in turbulence, Lecture Notes in Math, 898, 366-381, 1981.

Yang, W. and Tse, P.: Development of an advanced noise reduction method for vibration analysis based on singular value decomposition, NDT\&E international, 36, 419-432, 2003. 